\title{
Detection of Periodic Signals Using a New Adaptive Line Enhancer Based on Singular Spectrum Analysis
}

\author{
Saeid Sanei \\ Department of Computing \\ Faculty of Engineering and Physical Sciences, \\ University of Surrey, UK, \\ e-mail: s.sanei@surrey.ac.uk
}

\author{
Tracey K. M. Lee \\ School of Electrical and Electronic Engineering, Singapore \\ Polytechnic, Singapore and \\ School of Information Technology, Monash University, \\ Sunway Campus, Malaysia \\ e-mail: tlee@sp.edu.sg
}

\begin{abstract}
A new ALE-based on singular spectrum analysis (SSA) is proposed here. In this approach in the reconstruction stage of SSA the eigentriples are adaptively selected using the delayed version of the data. Unlike for the conventional ALE where order statistics are taken into account, here full eigen-spectrum of the embedding matrix is exploited. Consequently, the system works for non-Gaussian noise and wideband periodic signals. The performance of the system is demonstrated for synthetic as well as real signals and compared with those of traditional ALE.
\end{abstract}

Keywords; SSA; ALE; Sparsity

\section{INTRODUCTION}

Many signals and time series in nature have periodic or cyclo-stationary components. These signals are often buried in noise or mixed with other periodic or non-periodic signals. Extraction of such cyclic activities is very important for monitoring the status of a system.

Separation of a low-level sinusoid or narrow-band signal from broad-band noise has been a classical problem and ALE was introduced for this purpose by Widrow et al. [1]. The general block diagram of the ALE is depicted in Figure 1. The ALE input $s(t)$ is assumed to be the sum of a narrowband signal $x(t)$, and a broad-band signal $n(t)$. The parameters of the prediction filter $\mathbf{w}$ are adapted in such a way that the statistical mean squared error (MSE), $\mathrm{E}\left[e^{2}(t)\right]$, where E[.] stands for expectation, is minimized. The ALE operates by virtue of the difference between the correlation lengths of $x(t)$ and $n(t)$. The delay parameter $\Delta$ should be chosen larger than the correlation length of $n(t)$, but smaller than the correlation length of $x(t)$. In this case, it is possible for $\mathbf{w}$ to make a $\Delta$-step ahead prediction of $x(t-\Delta)$ based on the present and past samples of $s(t-\Delta)$. However, $\mathbf{w}$ is not able to predict $n(t)$ from knowledge about present and past samples of $n(t-\Delta)$. As a result, after the parameters of $\mathbf{w}$ have converged towards their optimal values, the error signal $e(t)$ is approximately equal to $n(t)$ and the ALE output $\hat{x}(t)$ is approximately equal to $x(t)$. Although the ALE is an effective tool for single channel signal denoising, it uses order statistics of the data within its minimization criterion. Moreover, its application is limited to narrowband

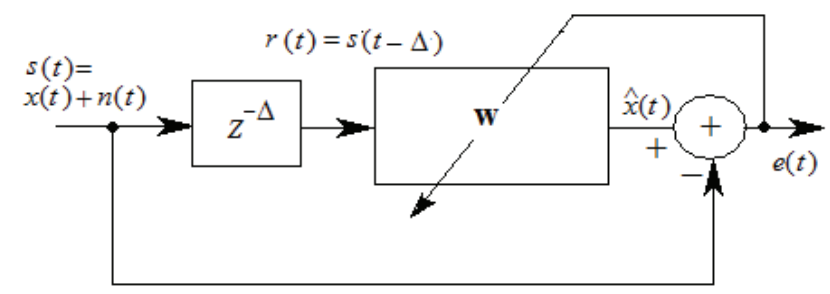

Fig. 1. Traditional adaptive line enhancer

Gaussian noise. Therefore, it is not applicable in important situations where the noise is not white Gaussian or the artefact signals are temporally correlated components.

SSA is becoming an effective and powerful tool for time series analysis in meteorology, hydrology, geophysics, climatology, economics, biology, physics, medicine and other sciences where short and long, one-dimensional and multidimensional, stationary and nonstationary, almost deterministic and noisy time series are to be analyzed. A comparison between SSA and other classical methods can be seen in [3]. Here, we show that SSA can decompose the signal space into distinct subspaces. This concept is exploited in designing a new high performance ALE.

\section{SINGULAR SPECTRUM ANALYSIS}

The basic SSA method consists of two complementary stages: decomposition and reconstruction. In the first step the series is decomposed and in the second stage the original series is reconstructed and used for further analysis. The main concept in studying the properties of SSA is separability, which characterizes how well different source signals can be separated from their single channel mixtures. To cope with the absence of approximate separability there 
are different ways of modifying SSA leading to different versions such as SSA with single and double centering, Toeplitz SSA, and sequential SSA [2]. A brief description of the two SSA stages is given in the following subsections.

Decomposition-This stage includes an embedding operation followed by singular value decomposition (SVD). It maps a one-dimensional time series $\mathbf{f}$ into a $k \times l$ matrix with rows of length $l$ as

$$
\begin{aligned}
\mathbf{H} & =\left[\mathbf{s}_{1}, \mathbf{s}_{2}, \ldots, \mathbf{s}_{k}\right] \\
& =\left[\begin{array}{ccccc}
f_{0} & f_{1} & f_{2} & \ldots & f_{k-1} \\
f_{1} & f_{2} & f_{3} & \ldots & f_{k} \\
f_{2} & f_{3} & f_{4} & \ldots & f_{k+1} \\
\vdots & \vdots & \vdots & \ddots & \vdots \\
f_{l-1} & f_{l} & f_{l+1} & \ldots & f_{r-1}
\end{array}\right]
\end{aligned}
$$

with vectors (segments of the original data $s(t)) \mathbf{s}_{i}=\left[f_{i-1}, f_{i}\right.$. $\left.\ldots, f_{i+l-2}\right]^{T} \in R^{l}$, where $k=r-l+1$ is the window length $(1 \leq l$ $\leq r$ ), and subscript $T$ denotes the transpose of a vector. Vectors $\mathbf{s}_{i}$ are called l-lagged vectors (or, simply, lagged vectors). The trajectory matrix $\mathbf{H}$ is a Hankel matrix, i.e. all the elements along the diagonals $i+j=$ const. are equal.

In the SVD stage the SVD of the trajectory matrix is computed and represented as the sum of rank-one biorthogonal elementary matrices. Consider the eigenvalues of the covariance matrix $\mathbf{C}_{\mathrm{x}}=\mathbf{H} \mathbf{H}^{T}$ are denoted by $\lambda_{1}, \ldots, \lambda_{l}$ in decreasing order of magnitude $\left(\lambda_{1} \geq \ldots \geq \lambda_{l} \geq 0\right)$ and the corresponding orthogonal $r \times 1$ eigenvectors by $\mathbf{u}_{1}, \ldots, \mathbf{u}_{l}$. Set $d=\max \left(i\right.$; such that $\left.\lambda_{i}>0\right)=\operatorname{rank}(\mathbf{H})$ : If we denote $\mathbf{v}_{i}=\mathbf{H}^{T} \mathbf{u}_{i} / \sqrt{\lambda_{i}}$, where $\mathbf{v}_{\mathrm{i}}$ s are $1 \times r$, then, the SVD of the trajectory matrix can be written as:

$$
\mathbf{H}=\sum_{i=1}^{d} \mathbf{H}_{i}=\sum_{i=1}^{d} \sqrt{\lambda_{i}} \mathbf{u}_{i} \mathbf{v}_{i}^{T}
$$

if $\lambda_{1}>\lambda_{2}$, the information energy will be mostly concentrated in the most dominant matrix $\quad \mathbf{H}_{1}=\sqrt{\lambda_{1}} \mathbf{u}_{1} \mathbf{v}_{1}$ associated with the first few singular values). However, this is not always the case and an intelligent technique should be developed to accurately select the desired eigenvalues and the corresponding eigenvectors.

Reconstruction-During reconstruction the elementary matrices are first split into several submatrices, often called groups. The size of each group or in other words, the length of each subspace may be specified based on some a priori information. Let $I=\left\{i_{1}, \ldots, i_{p}\right\}$ be the indices corresponding to the $p$ eigenvalues of the desired component. Then, the matrix $\hat{\mathbf{H}}_{I}$ corresponding to the group $I$ is defined as
$\hat{\mathbf{H}}_{I}=\sum_{j=i_{1}}^{i_{p}} \mathbf{H}_{j}$. In splitting the set of indices $J=\{1, \ldots$, d) into disjoint subsets $I_{1}$ to $I_{m}$ we always have

$$
\mathbf{H}=\sum_{j=I_{1}}^{I_{m}} \hat{\mathbf{H}}_{j}
$$

Selection of the sets $I_{1}, \ldots I_{m}$ is called eigentriple grouping. For a given group $I$ the contribution of the component $\mathbf{H}_{I}$ in expansion (3) is measured by contribution of the corresponding eigenvalues: $\sum_{i \in I} \lambda_{i} / \sum_{i=1}^{d} \lambda_{i}$. Next, the obtained matrix is transformed to the form of a Hankel matrix which can be subsequently converted to a time series. If $z_{i j}$ stands for an element of a matrix $\mathbf{Z}$, then the $k$-th term of the resulting series is obtained by averaging $z_{i j}$ over all $i, j$ such that $i+j=k+1$ (Hankelization). In the above case the time series can be reconstructed from $\mathbf{H}$ in (3).

\section{SSA-BASED ALE}

In order to apply SSA for separation or denoising the signals, the corresponding subspace of the desired signal should be identified. This is probably the main shortcoming of basic SSA. In some very recent applications such as [4], [5], [6], [7] or [8] selection of periodic components has been decided by clustering the eigentriples [5] or establishing some criteria [6], [7], [8]. Such criteria can be set only if the periodic signal is narrow band or well defined. Even then, the signal may not be fully reconstructed and some error is often involved.

For the case of periodic signals, given the period, the information can be used in a way similar to that used in ALE. A delayed (by one period) version of the signal is then used as a reference for adaptive reconstruction of the signal. Unlike for basic SSA, where the eigenvalues of the desired signals have to be selected manually, here, the algorithm is adaptive and the signal periodicity is fully exploited (as for the ALE). A block diagram of the overall SSA-based ALE is depicted in Figure 2.

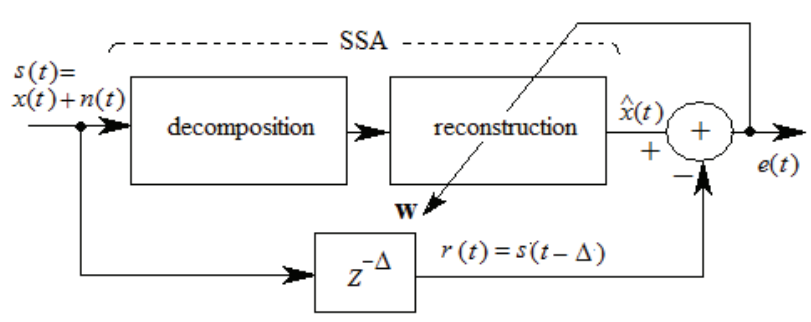

Fig. 2. Block diagram of the proposed SSA-based ALE

Assume the Hankel matrix for $r(t)=s(t-\Delta)$ is $\mathbf{R}$. During reconstruction the following cost function is minimised in order to make sure the correct subgroup of eigentriples is selected for reconstruction of the periodic signal. 


$$
J(\mathbf{W})=\left\|\mathbf{R}-\mathbf{U} \mathbf{W} \boldsymbol{\Lambda}^{\frac{1}{2}} \mathbf{V}^{T}\right\|_{F}^{2}
$$

where $\|\cdot\|_{F}$ denotes Frobenius norm. $\mathbf{U}, \Lambda$ (diagonal matrix of eigenvalues), and $\mathbf{V}$ are the SVD factors (as in eq. (2)) and $\mathbf{W}$ is a $l \times l$ diagonal matrix of adaptive weights $w_{\mathrm{ij}}$. Its size is the same as $\Lambda$ 's. To minimise this cost function a simple gradient approach leads to the following update equation:

$$
\mathbf{W}_{k+1}=\mathbf{W}_{k}-\mu \mathbf{U} \Lambda^{\frac{1}{2}} \mathbf{V}^{T}\left(\mathbf{R}-\mathbf{U} \mathbf{W}_{k} \Lambda^{\frac{1}{2}} \mathbf{V}^{T}\right)^{T}
$$

Where $\mu$ is the step size (which is often set manually but can be adapted to the convergence rate). The weights of the filter, $\mathbf{W}$, are estimated using (5). In the reconstruction process $\mathbf{W}$ is multiplied by $\Lambda^{1 / 2}$ and the desired signal is recovered during the SSA reconstruction process.

Another obvious information about the eigenspectrum is its sparsity since the periodic signal eigenvalues have often very small number of non-zero components. In the following section this information is exploited.

\section{SPARSITY CONSTRAINT}

The weighted eigenvalue pattern for periodic signals is expected to have a small number of non-zero components. This concept can be exploited and used to further improve the optimization algorithm. To maximise the sparsity, the number of non-zero components (weighted eigenvalues) should be minimised. This is equivalent to minimising the $\mathrm{L}_{0}$-norm of $\mathbf{W} \Lambda^{1 / 2}$. Since this quantity is NP hard, it is often approximated by the $\mathrm{L}_{1}$-norm. The two costs can be combined to change the above constrained problem into an unconstrained one using a penalty term. Therefore, the overall cost function can be written as:

$$
J(\mathbf{W})=\left\|\mathbf{R}-\mathbf{U} \mathbf{W} \mathbf{\Lambda}^{\frac{1}{2}} \mathbf{V}^{T}\right\|_{F}^{2}+\rho\left\|\operatorname{vec}\left(\operatorname{diag}\left(\mathbf{W} \mathbf{\Lambda}^{\frac{1}{2}}\right)\right)\right\|_{l_{1}}
$$

or

$$
J(\mathbf{W})=\left\|\mathbf{R}-\mathbf{U} \mathbf{W} \Lambda^{\frac{1}{2}} \mathbf{V}^{T}\right\|_{F}^{2}+\rho\left\|\operatorname{vec}\left(\boldsymbol{\Lambda}^{\frac{1}{2}}\right) \mathbf{W}\right\|_{l_{1}}
$$

where $\|\cdot\|_{l_{1}}$ denotes $\mathrm{L}_{1}$-norm, vec(B) assigns the diagonal elements of $1 \times d \mathrm{~B}$ to a vector of size $1 \times d$, and $\rho$ is the penalty term which is a fixed Lagrange multiplier. Thus, the new update equation for estimating $\mathbf{W}$ can be achieved as:

$$
\begin{aligned}
\mathbf{W}_{k+1}= & \mathbf{W}_{k}-\mu \mathbf{U} \mathbf{\Lambda}^{\frac{1}{2}} \mathbf{V}^{T}\left(\mathbf{R}-\mathbf{U} \mathbf{W}_{k} \mathbf{\Lambda}^{\frac{1}{2}} \mathbf{V}^{T}\right)^{T} \\
& +\rho^{\prime} \cdot \operatorname{sign}\left[\mathbf{W}_{k} \operatorname{vec}\left(\boldsymbol{\Lambda}^{\frac{1}{2}}\right)\right] \cdot\left(\operatorname{vec}\left(\boldsymbol{\Lambda}^{\frac{1}{2}}\right)\right)^{T}
\end{aligned}
$$

Where $(.)^{T}$ stands for transpose operation, $k$ is the iteration index, and $\rho^{\prime}=\mu \cdot \rho$. The best initialization for $\mathbf{W}$ is the identity matrix $\mathbf{I}$ since $\mathbf{W}$ is expected ultimately to be a diagonal matrix. This is computed for each block of data separately. However, the recursive algorithm in [5] can also be used for real time processing of the data.

\section{COMPARING BASIC ALE AND SSA-ALE}

To compare the new ALE with the traditional ALE, a number of experiments are carried out using both synthetic and real signals. For the synthetic data a noisy sinusoid is generated. For real data a surface electromyography (EMG) signal is used. This signal is corrupted by an electrocardiogram (ECG) signal. Therefore, the problem here can be considered as recovering of the ECG from strong noise-like EMG background or other way round. The results are presented for each experiment.

\section{A. Simulated signals}

A four second, $N=2000$ sample sinusoid of $500 \mathrm{~Hz}$ and 0.1 volt amplitude is generated. In one experiment Gaussian noise and in another one impulsive noise are added. The input SNR is varied between -10 to $+10 \mathrm{~dB}$ and both the traditional ALE and the new proposed SSA-based ALE have been applied to the signals. Figure 3 presents the performance of the two ALEs in terms of output SNR. The output SNR is defined as:

$$
S N R_{\text {out }}=\frac{\sum_{t=1}^{N} \hat{x}^{2}(t)}{\sum_{t=1}^{N}(x(t)-\hat{x}(t))^{2}}
$$

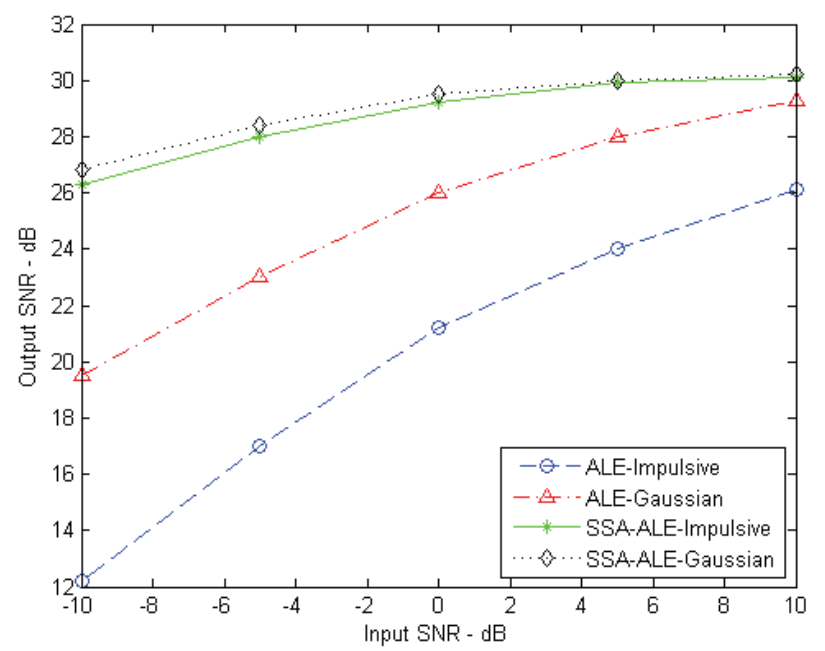

Fig. 3. Output SNRs for different input SNRs for synthetic data for both traditional ALE and SSA-based ALE methods. where $\hat{x}(t)$ is the estimated desired signal. It is seen that, given a narrowband signal (sinusoid) for Gaussian noise, the performances of both methods are close to each other. 
However, when the noise is heavy tailed, the performance of the SSA-based ALE is considerably better than that of the ALE. Figure 3 shows the outputs for an input $S N R=-5 d B$. The results are the average results over 10 segments of similar recordings from 5 subjects.

\section{B. Real signals (EMG corrupted by ECG artifact)}

EMG signals provide valuable information relating to peripheral and central motor functions and have been widely adopted in the study of motor function and movement disorders. Surface EMGs represent a superposition of electrical activities from motor unit action potentials located subcutaneous to the detecting electrodes.
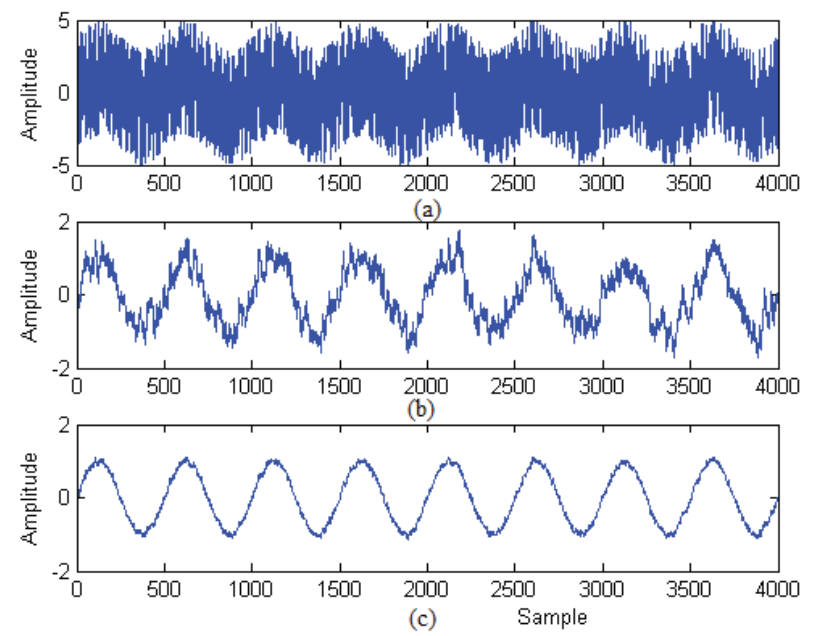

Fig. 4. (a) the synthetic noisy signal with an impulsive noise of $-5 \mathrm{~dB}$ SNR, (b) output of traditional ALE, and (c) output of the proposed SSA-based ALE.

These signals however are nonstationary and often available using only single-channel recordings. Most of EMG signals are corrupted by ECG mainly because blood vessels are spread all over the body. Restoration of EMG signals from artefacts is extremely difficult mainly due to their randomness and being noisy in nature. There have been some attempts in denoising the EMG signals recently such as that in [9] where wavelet and ICA have been used for a multichannel recording of EMG. Unfortunately, having more than one channel recording is not always possible. Moreover, generally the conditions of independency and stationarity of the signal components are not fully satisfied. In a very recent attempt by $\mathrm{Lu}$ et al. [10] an adaptive noise canceller (ANC) based on recursive-least-squares (RLS) algorithm was developed for removing ECG artefact from surface EMGs recorded in patients with cervical dystonia. Even in this approach an ECG signal recorded from a separate channel was used as a reference signal. However, so far, to the authors' knowledge, no prior attempts have been made to separate noisy ECG and EMG signals from a single channel recording without a reference component.

An exhaustive search may be carried out over the eigenvalues and finally select a small number of eigentriples which belong to the ECG signal and reconstruct the ECG from them. In such cases some criteria have to be established for selection of the eigentriples of the desired signals. However, in many cases such as when the EMG is stronger than the ECG, this method won't be accurate and is computationally demanding. On the other hand, using ALE is not fully justified since the noise (EMG) distribution is not known and it is often heavy tailed. Therefore, for the above reasons, adaptive selection of the SSA eigentriples, followed in this paper, is the most logical approach.

The EMG signals were recorded from the human forearm using surface EMG. The sampling frequency was $2 \mathrm{KHz}$ and the subject was relatively relaxed during slow arm movements. The overall recorded data length was 70 seconds (140Kdata samples), divided into $20 \mathrm{~K}$ sample segments (10 sec each). In these experiments no preprocessing was performed. Therefore, the noisy EMG remained un-altered and the ECG was completely separated.

The period of ECG, heart rate, can be measured manually. Other techniques, such as analyzing the autocorrelation function (ACF) can also be used. ACF is often used for detecting the pitch period in speech signals. Fortunately, in this new application of SSA there is no need for selection of the number of desired eigentriples (window length) since the eigentriples are automatically weighted by the estimated $\mathbf{W}$. The only setting here is the overall number of eigentriples which can vary over a wide range without any noticeable change in the results. In this experiment we selected this number to be $d=240$.

Both traditional ALE and the proposed SSA-based ALE are examined here. The data were obtained from 21 subjects each recorded two times within approximately one hour time intervals. However, for illustration, only one segment of one record from one of the subjects (selected randomly with no preference) was taken. Figure 5 shows the results of applying traditional ALE, proposed SSA-based ALE, and finally when sparsity constraint is added for the same signal segment. In this figure the original segment is shown in the top row. What is very important here is that the separated ECG using the proposed methods (with or without sparsity constraint) can be directly used for clinical diagnosis. For the traditional ALE the separated ECG is not of sufficient quality to be used as an indicator of heart function. Finally, the concept in Section 4 was applied to impose the sparsity constraint. Since the primary objective in separation is to have independency or uncorrelatedness between the output components, the correlation coefficients of both normalized 

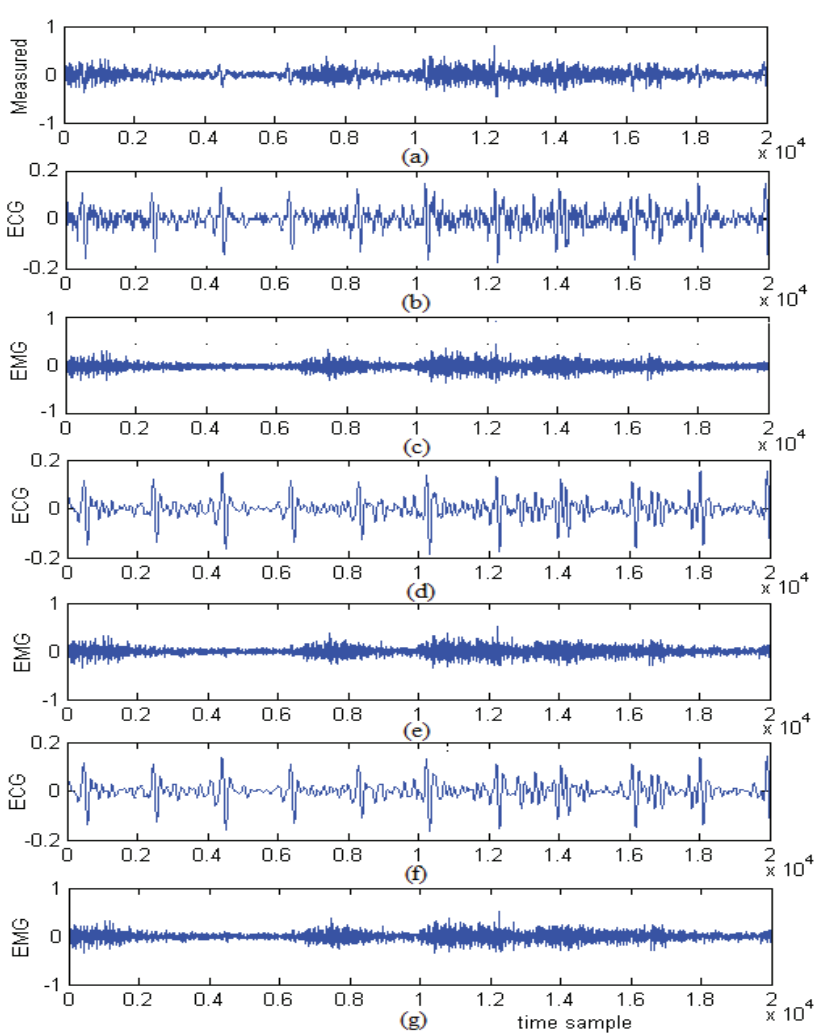

Fig. 5. Separation of ECG and EMG signal in (a) using (b),(c) the traditional ALE, (d),(e) the proposed SSA-based ALE, and (f),(g) when the sparsity constraint is added to the SSA-based ALE.

separated signals, $\xi=\frac{1}{N} \sum_{i=1}^{N} E M G(i) \cdot E C G(i), N=$ 140000, are calculated. As depicted in Table 1, the value of this coefficient for the traditional ALE output is $\xi_{\mathrm{ALE}}=$ 0.079 , whereas for the output of the SSA-based ALE this coefficient is negligible $\left(\xi_{\text {SSA-ALE }}=0.0016\right)$.

TABLE I. Correlation coefficient ( \pm max variation) between the estimated ECG and EMG for 42 data segments from 21 subjects using both traditional ALE and the SSA-based ALE methods.

\begin{tabular}{c|c|c|c}
\hline & $\begin{array}{c}\text { Traditional } \\
\text { ALE }\end{array}$ & SSA- ALE & $\begin{array}{l}\text { SSA-ALE } \\
+ \text { sparsity } \\
\text { constraint }\end{array}$ \\
\hline$\xi$ & $0.079 \pm 0.0022$ & $0.0018 \pm 0.0003$ & $0.0016 \pm 0.0003$
\end{tabular}

\section{CONCLUDING REMARKS}

A new ALE based on SSA has been designed here. It outperforms traditional ALE due to three major reasons; (1) unlike in traditional SSA, the estimation of the filter coefficients is based on the full spectrum of SSA eigenvalues, (2) the noise doesn't need to be stationary or Gaussian, and (3) the periodic signal can be wideband.
Moreover, there is no need for accurate setting of the SSA parameters. The signal components have distinct subspaces. Therefore, the method is more effective ad efficient than the traditional ALE. The proposed system is more robust against noise than the traditional ALE. It is also concluded that incorporating sparsity constraint leads to further but negligible improvement of the SSA-based ALE.

\section{REFERENCES}

[1] B. Widrow, J. R. Glover, H. M. McCool, J. Kaunitz, C. S. Williams, R. H. Hearn, J. R. Zeidler, E.U. Dong, and R. C. Goodlin, "Adaptive noise cancelling: principles and applications," Proc. IEEE, Vol. 63, pp. 1692-1719, 1975.

[2] N. Golyandina, V. Nekrutkin, and A. Zhigljavsky, Analysis of time series structure: SSA and related techniques, Chapman \& Hall/CRC, New York 2001.

[3] T. H. Alexandrov and N. Golyandina, "The automatic extraction of time series trend and periodical components with the help of the Caterpillar-SSA approach," Exponenta Pro 3-4, pp. 54-61, 2004.

[4] M. Ghodsi, H. Hassani, and S. Sanei, "Extracting fetal heart signal from noisy maternal ECG by singular spectrum analysis," Journal of Statistics and its Interface, Special Issue on the Appl. of SSA, vol. 3, pp. 399-411, 2010.

[5] A. R. Teixeira, A. M. Tomé, M. Böhm, C. G. Puntonet, and E. W. Lang, "How to apply nonlinear subspace techniques to univariate biomedical time series," IEEE Trans. on Instrumentation and Measurement, Vol. 58, No. 8, PP. 2433-2443, 2009.

[6] S. Sanei, M. Ghodsi, and H. Hassani, "A constrained singular spectrum analysis approach to murmur detection from heart sounds," Elsevier Journal of Medical Engineering and Physics, Vol. 33, Issue 3, PP. 362-367, 2011.

[7] S. Sanei and A. R. Hosseini-Yazdi, "Separation of single channel EMG and ECG signals using constrained singular spectrum analysis," Proc. of the $17^{\text {th }}$ Int. Conf on Digital Signal Processing (DSP), Corfu Greece, 2011.

[8] F. Ghaderi, H. R. Mohseni, and S. Sanei, "Localizing heart sounds in respiratory signals using singular spectrum analysis," IEEE Trans. on Biomed. Eng. 2011, in Press.

[9] B. Azzerboni, M. Carpentieri, F. L. Foresta, and F. C. Morabito, Neural-ICA and wavelet transform for artifacts removal in surface EMG, Proc. Int. Joint Conf. Neural Networks, pp. 3223-3228, 2004.

[10] G. Lu, J.-S. Brittain, P. Holland, J. Yianni, A. L. Green, J. F. Stein, T. Z. Aziz, and S. Wang, "Removing ECG noise from surface EMG signals using adaptive filtering," Neuroscience Letters, vol. 462 pp. 14-19, 2009. 